\title{
sPHENIX TPC simulation studies
}

\section{Sourav Tarafdar*, for sPHENIX collaboration}

Vanderbilt University, Nashville, TN, U.S.A

E-mail: sourav.tarafdarevanderbilt.edu

Proposed upgrade of PHENIX to sPHENIX at RHIC is focused on measuring jets, jet correlations and three states of upsilons to determine the temperature dependence of transport coefficients of the quark-gluon plasma and complementing measurements being made at LHC. The sPHENIX detector will have Gaseous Electron Multiplier (GEM) based Time Projection Chamber (TPC) as an outer tracking detector with a length of $211 \mathrm{~cm}$ and outer radius of $78 \mathrm{~cm}$ spanning phase space of full azimuth and 2.2 units in pseudo rapidity. Space charge due to the accumulation of less mobile positive ions within TPC volume is considered one of the important factor determining the performance of GEM-based TPC in Heavy Ion collision environment. Also, selection of suitable gas mixture is important to achieve high mobility of ionized electrons and ions within TPC gas volume. This article is intended to present the simulation of the effect of space charge and diffusion coefficients of different gas mixtures in TPC on tracking performance.

5th International Conference on Micro-Pattern Gas Detectors (MPGD2017)

22-26 May, 2017

Philadelphia, USA

${ }^{*}$ Speaker. 


\section{Tracking Physics Motivation}

One of the main objective for sPHENIX[1] is to measure well resolved all the three upsilon states $(\Upsilon(1 s), \Upsilon(2 s), \Upsilon(3 S))$. Each of the Upsilon state has different radii and binding energy and hence measuring all three states of Upsilons will enable us to compare the effect of medium produced in Heavy Ion Collisions at RHIC simultaneously on three bottominium states. Both PHENIX[2] and STAR[3] has measured in-medium modification of combined three $\Upsilon$ states. However the mass-resolved $\Upsilon$ states measurement in proposed sPHENIX will be important in terms of understanding the difference in RHIC and LHC color screening environment[4], providing experimental verification about theoretical prediction of higher coalescence for $\Upsilon$ at $\mathrm{LHC}$ as compared to RHIC[5]. The direct comparison between $J / \psi$ in medium modifications and $\Upsilon(2 S)$ states at RHIC energy will also be possible with the propsed sPHENIX $\Upsilon$ measurment.

Another Physics goal for sPHENIX is to measure fragmentation functions[6] via charged track measurements independent of measurement via calorimentric jet energy. This requires charged tracks to be coincident with a high energy jet reducing the fake track contribution. The proposed measurement of the fragmentation function at RHIC is important after surprising results from ATLAS[7] and CMS[8].

Both measurements of all three $\Upsilon$ states and fragmentation function requires tracking detectors with large acceptance, high rate data acquisition, good momentum resolution with high track reconstruction efficiency. For resolving three states of $\Upsilon$ the tracker must have momentum resolution of $1.2 \%$ for $4<p_{T}<10 \mathrm{GeV} / c$ with mass resolution of $\sim 100 \mathrm{MeV}$ and track reconstruction efficiency $>90 \%$. Fragmentation function measurement also requires excellent tracking resolution upto $40 \mathrm{GeV} / \mathrm{c}$ in $p_{T}(d p / p<20 \% \times \mathrm{p})$.

The tracking configuration of the proposed sPHENIX detector consists of Time Projection Chamber (TPC) which alongwith other tracking susbsystem will play pivotal role in acheiving the required tracking performance.

\section{SPHENIX TPC}

The sPHENIX tracker configuration consists of 3-layers of MAPS pixel inner barrel (MVTX with $|\eta|<1.1$ and $\Delta \Phi=2 \pi$ ), 4-layer silicon strip intermediate tracker (INTT with $|\eta|<1.1$ ) and TPC outer tracker with $|\eta|<1.1$ and $\Delta \Phi=2 \pi$. The design of TPC follows cylindrical layout with membrane electrode located at the middle of interaction region dividing the TPC volume into two symmetrical TPC gas volume. The membrane electrode is set to high voltage bias while the readout plane for each TPC volume, located on the endcap inner surface facing the gas volume, is set at ground potential. The difference in potential in either volume of the TPC provides the necessary electric field for transporting primary ionization to the readout plane. In order to maintain uniform electric drift field from central membrane to readout modules the gas volume of TPC is surrounded by a series of conducting rings held at uniformly decreasing potential by precision-matched resistor chain known as field cage. TPC amplification element is provided by 4 layers of GEM detectors[9] and is directly coupled to the readout plane on the inner side of TPC volume. Operation of GEM causes Ion Back Flow (IBF) and can contribute to the accumulation of space charge within the TPC gas volume which distorts track reconstruction. So it is imperative to suppress IBF which can be 
done by proper choice of gas and also selecting the initial point of tracking within TPC volume. The following section shows some simulation results for optimizing TPC tracking performance.

\section{TPC tracking simulation}

In order to tune TPC parameters 500 pure pions per event with $0.2<p_{T}<40 \mathrm{GeV} / \mathrm{c}$ generated within phase space of $|\eta|<1.1$ and $\Delta \Phi=2 \pi$ are passed through sPHENIX Geant4 detector geometry with all the tracking detectors in active state and then through sPHENIX track reconstruction algorithm. After reconstruction of the tracks, $p_{T}$ resolution and single track efficiency were calculated to determine the performance of tuned TPC.

\subsection{Effect of space charge on TPC tracking}

Left panel of fig. 1 shows the distortion in track due to space charge accumulation within different active TPC gas volume. Evidently there is substantial decrease in track distortion due to space charge if the inner radius of field cage of TPC is kept at $20 \mathrm{~cm}$ and the tracking starts from $30 \mathrm{~cm}$. With this configuration there is no effect of space charge on $p_{T}$ resolution (middle panel of fig. 1) and also on single track efficiency (right panel of fig. 1).


Figure 1: Track distortion as a function of $\mathrm{R}$ within TPC gas volume for different inner radius of field cage (left panel). Comparison of $p_{T}$ resolution (middle panel) and single track efficiency (right panel) as a function of true $p_{T}$ for 500 pions/event with and without space charge when the tracking starts from $30 \mathrm{~cm}$ from beam axis inside TPC active volume.

\subsection{Effect of changing TPC readout dimension}

After determining the starting point of tracking within TPC volume few studies were done to estimate the final point of tracking. This involve changing the outer radius of the readout plane of TPC while keeping the starting point of tracking at around $30 \mathrm{~cm}$. Figure 2 shows the tracking performance in terms of single track efficiency and $p_{T}$ resolution for 500 pions/event. Changing the starting point of tracking from $30 \mathrm{~cm}$ to $31.5 \mathrm{~cm}$ don't affect the tracking performance as seen in fig. 2. However the tracking performance deteriorates if the tracking is done only upto $75 \mathrm{~cm}$. The tracking performace remains the same if the tracking within TPC is terminated at $78 \mathrm{~cm}$ instead of $80 \mathrm{~cm}$. The outer radius of the TPC readout is set at $78 \mathrm{~cm}$ from beam axis which concludes the study of keeping the tracking within TPC for $30 \mathrm{~cm}<\mathrm{R}<78 \mathrm{~cm}$. 



Figure 2: $p_{T}$ resolution (left panel) and single track efficiency (right panel) as a function of true $p_{T}$ with 500 pions/event for different outer radii of TPC readout. The different colored lines in $p_{T}$ resolution plot represents the fit function $\Delta p_{T} / p_{T}=\sqrt{A^{2}+B^{2} p_{T}^{2}}$ where $\mathrm{A}$ and $\mathrm{B}$ are fit parameters.

\begin{tabular}{cccc}
\hline Gas & Drift velocity & Transverse Diffusion & Longitudinal Diffusion \\
\hline NeCF4(90:10) & $80 \mathrm{um} / \mathrm{sec}$ & $64 \mathrm{um} / \sqrt{\mathrm{cm}}$ & $120 \mathrm{um} / \sqrt{\mathrm{cm}}$ \\
NeCF4(94:6) & $70 \mathrm{um} / \mathrm{sec}$ & $80 \mathrm{um} / \sqrt{\mathrm{cm}}$ & $160 \mathrm{um} / \sqrt{\mathrm{cm}}$ \\
\hline
\end{tabular}

Table 1: Neon based gas parameters at $400 \mathrm{~V} / \mathrm{cm}$ and $1.4 \mathrm{~T}$.

\subsection{Tracking performance using different gas in TPC}

Selecting gas for TPC is important in reducing space charge, central membrane operating potetial selection, single point resolution improvement and multiple scattering reduction. As sPHENIX TPC is based on avalanche mechanism so the spread of avalanche across the readout pads relies on diffusion of charge. Furthermore high ion mobility of gas helps to reduce the accumulation of space charge within TPC gas volume. Considering these factors Neon based gas are being considered to be used in TPC. Table 1 shows the gas parameters for two variant of Neon based gas mixture which has drift velocity in the pleateu region at the TPC operating voltage of $400 \mathrm{~V} / \mathrm{cm}$ and 1.4T magnetic field. Fig. 3 shows the tracking performance for NeCF4(90:10) and NeCF4(94:6). Both NeCF4(90:10) and NeCF4(94:6) has almost the same single track efficiency. However, NeCF4(94:6) has slightly better $p_{T}$ resolution for $p_{T}>5 \mathrm{GeV} / c$ still NeCF4(90:10) has been considered as better option for $\mathrm{dE} / \mathrm{dx}$ measurement.
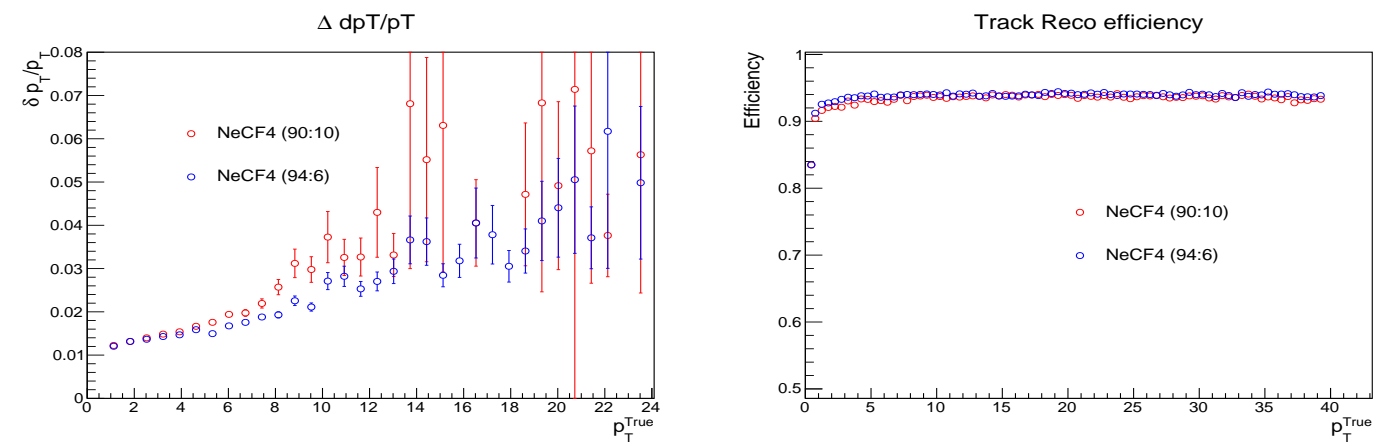

Figure 3: $p_{T}$ resolution (left panel) and single track efficiency (right panel) with 500 pions/event for NeCF4(90:10) and NeCF4(94:6) gas mixtures. 


\section{Conclusion}

Various simulation studies to optimize the tracking performance of sPHENIX TPC has been shown in this article. The $p_{T}$ resolution of the sPHENIX tracking configuration is good enough to resolve the three upsilon states. Fig. 4 shows the sPHENIX Geant 4 simulation of upsilon mass spectra with upsilon embedded in most central Au+Au HIJING events at $\sqrt{S_{N N}}=200 \mathrm{GeV}$ using the proposed tracking configuration. The mass resolution of upsilon 1 s state is about $92 \mathrm{MeV} / \mathrm{c}^{2}$ and it is evident that TPC alongwith the other tracking detectors will be able to resolve all the three states of upsilon.

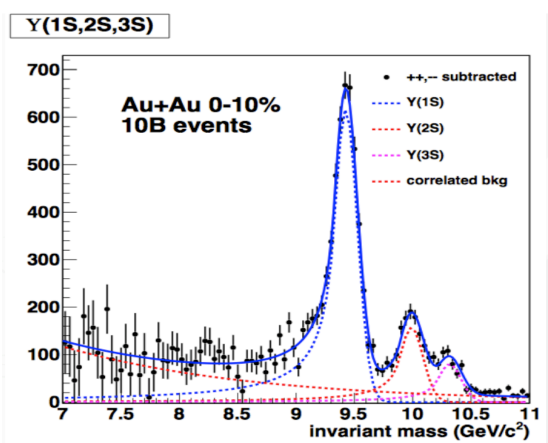

Figure 4: Geant4 simulated mass spectra of upsilon embedded in $\mathrm{b}=0.4 \mathrm{fm} A u+A u$ HIJING events using sPHENIX tracking detectors.

\section{References}

[1] A. Adare et al., An Upgrade Proposal from the PHENIX Collaboration, 2014, arXiv:1501.06197.

[2] A. Adare et al., Measurement of $\Upsilon(1 S+2 S+3 S)$ production in $p+p$ and Au+Au collisions at $\sqrt{s_{N N}}=200 G e V$, Phys. Rev. C91(2015) 024913,[arXiv: 1404.2246$]$.

[3] L. Adamczyk et al., Suppression of Upsilon Production in $d+A u$ and $A u+A u$ Collisions at $\sqrt{s_{N N}}=200 \mathrm{GeV}$, Phys. Lett. B735(2014) 127,[arXiv:1312.3675].

[4] M. Habich et al., Particle spectra and HBT radii for simulated central nuclear collisions of $C+C$, $A l+A l, C u+C u, A u+A u$, and $P b+P b$ from $\sqrt{s}=62.4-2760 \mathrm{GeV}, 2014$, Eur. Phys. J. C(2015) 75:15,[arXiv:1409.0040].

[5] A. Emerick et al., Bottomonia in the quark-gluon plasma and their production at RHIC and LHC, 2011, Eur. Phys. J. A(2012) 48:72,[arXiv:1111.6537].

[6] K. Nakamura et al., Review of Particle Physics (PDG), JPG 37 (075021) 2010.

[7] G. Aad et al., Measurement of inclusive jet charged-particle fragmentation functions in $\mathrm{Pb}+\mathrm{Pb}$ collisions at $\sqrt{s_{N N}}=2.76 \mathrm{TeV}$ with the ATLAS detector, 2014, Phys. Lett. B739(2014) 320,[arXiv:1406.2979].

[8] Serguei Chatrchyan et al., Measurement of jet fragmentation in PbPb and pp collisions at $\sqrt{\left(s_{N N}\right)}=$ 2.76 TeV, Phys. Rev., C90:024908, 2014, [arXiv: 1406.0932 ].

[9] F. Sauli, GEM: A new concept for electron amplification in gas detectors,Nucl. Instr. and Meth. A $\mathbf{3 8 6}$ (1997) 531. 